OPEN ACCESS

Edited by:

Jordi Giralt,

Vall d'Hebron University Hospital,

Spain

Reviewed by:

Olga Hamming-Vrieze,

The Netherlands Cancer Institute

(NKI), Netherlands

Sergi Benavente,

Vall d'Hebron University Hospital,

Spain

*Correspondence:

Tairo Kashihara

kashiharatairo@gmail.com

Specialty section:

This article was submitted to Head and Neck Cancer,

a section of the journal

Frontiers in Oncology

Received: 09 January 2021

Accepted: 30 March 2021

Published: 27 April 2021

Citation:

Kashihara T, Nakamura S,

Murakami N, Ito $K$, Matsumoto $Y$, Kobayashi K, Omura G, Mori T, Honma Y, Kubo Y, Okamoto $H$,

Takahashi K, Inaba K, Okuma $K$,

Igaki $H$, Nakayama $Y$, Kato $K$, Matsumoto $F$, Yoshimoto $S$ and Itami J (2021) Initial Experience of Intentional Internal High-Dose Policy Volumetric

Modulated Arc Therapy of Neck

Lymph Node Metastases $\geq 2 \mathrm{~cm}$ in

Patients With Head and Neck

Squamous Cell Carcinoma.

Front. Oncol. 11:651409.

doi: 10.3389/fonc.2021.651409

\section{Initial Experience of Intentional} Internal High-Dose Policy Volumetric Modulated Arc Therapy of Neck Lymph Node Metastases $\geq 2 \mathrm{~cm}$ in Patients With Head and Neck Squamous Cell Carcinoma

\author{
Tairo Kashihara ${ }^{1 *}$, Satoshi Nakamura ${ }^{1}$, Naoya Murakami ${ }^{1}$, Kimiteru $_{\text {Ito }}{ }^{2}$, \\ Yoshifumi Matsumoto ${ }^{3}$, Kenya Kobayashi ${ }^{3}$, Go Omura ${ }^{3}$, Taisuke Mori ${ }^{4}$, \\ Yoshitaka Honma ${ }^{5}$, Yuko Kubo ${ }^{2}$, Hiroyuki Okamoto ${ }^{1}$, Kana Takahashi ${ }^{1}$, Koji Inaba ${ }^{1}$, \\ Kae Okuma ${ }^{1}$, Hiroshi Igaki ${ }^{1}$, Yuko Nakayama ${ }^{1}$, Ken Kato ${ }^{5}$, Fumihiko Matsumoto ${ }^{3}$, \\ Seiichi Yoshimoto ${ }^{3}$ and Jun Itami ${ }^{1}$ \\ ${ }^{1}$ Department of Radiation Therapy in National Cancer Center Hospital, Tokyo, Japan, ${ }^{2}$ Department of Radiology in National \\ Cancer Center Hospital, Tokyo, Japan, ${ }^{3}$ Department of Head and Neck Oncology in National Cancer Center Hospital, \\ Tokyo, Japan, ${ }^{4}$ Department of Pathology and Clinical Laboratories in National Cancer Center Hospital, Tokyo, Japan, \\ ${ }^{5}$ Department of Head and Neck Medical Oncology in National Cancer Center Hospital, Tokyo, Japan
}

Background and Purpose: Most locoregional recurrences after definitive radiotherapy for head and neck squamous cell carcinoma (HNSCC) develop "in-field." Dose escalation while sparing organs at risk can be a good solution for improving local control without increasing adverse effects. This study investigated the safety and effectiveness of volumetric modulated arc therapy (VMAT) using intentionally internal high-dose policy (IIHDP) to treat neck lymph node metastases (NLNM) $\geq 2 \mathrm{~cm}$ in HNSCC patients.

Materials and Methods: We analyzed 71 NLNM from 51 HNSCC patients who had received definitive radiotherapy to treat NLNM $\geq 2 \mathrm{~cm}$ using the VMAT technique in our institution between February 2017 and August 2019. Thirty-seven NLNM from 25 patients were treated using IIHDP VMAT (group A), and 34 NLNM from 27 patients were treated with homogeneous-dose distribution policy (HDDP) VMAT (group B). One patient with three NLNM had one lymph node assigned to group $A$ and the other two to group B. Adverse events and local recurrence-free survival (LRFS) was compared between the two groups.

Results: In the median follow-up period of 527 days, there were no significant differences between the groups in terms of dermatitis or mucositis $\geq$ grade $2 / 3$, but LRFS was significantly longer in group $A(p=0.007)$. In the Cox regression analysis after adjustment for the propensity score, group A also showed an apparently superior LFRS. 
Conclusion: Our initial experience of IIHDP VMAT suggested that IIHDP VMAT to treat HNSCC neck lymph node metastases measuring $\geq 2 \mathrm{~cm}$ was feasible and possibly led to better local control than HDDP VMAT.

Keywords: radiotherapy, volumetric modulated-arc therapy, simultaneous integrated boost, head and neck cancer, bulky tumors

\section{INTRODUCTION}

Head and neck cancer is common worldwide, with more than 800,000 new cases and 400,000 deaths annually (1). Intensitymodulated radiotherapy (IMRT) and volumetric modulated arc therapy (VMAT) are widely used to treat head and neck cancer because they are associated with fewer adverse effects than conventional 3-dimensional (3D) radiation therapy (2-6). On the other hand, in GORTEC 2004-01 randomized phase III trial of locally advanced head and neck squamous cell carcinoma (HNSCC) patients, locoregional control was not improved with dose-escalated IMRT compared with 70 Gy in 35 fractions (7).

In contrast, a previous study reported a patient with bulky uterine cervical cancer who showed complete response using brachytherapy with increasing radiation dose inside the tumor, while sparing organs at risk (OARs) (8). This technique was named "intentional internal high dose policy (IIHDP)" brachytherapy and could be a new strategy for enhancing local tumor control without increasing treatment-related adverse effects.

In our institution, IIHDP VMAT has been applied to increase the dose delivered to bulky neck lymph node metastases $\geq 2 \mathrm{~cm}$ in head and neck squamous cell carcinoma (HNSCC). In the present study, to investigate the safety and effectiveness of IIHDP VMAT to treat neck lymph node metastases of HNSCC, we retrospectively compared neck lymph node metastases $\geq 2 \mathrm{~cm}$ treated with and without IIHDP VMAT.

\section{MATERIALS AND METHODS}

\section{Patient Population}

The flowchart of patient inclusion is shown in Table 1. We conducted a retrospective review, from our institutional database, of the data of 394 patients with HNSCC who underwent radiotherapy using the VMAT technique to treat neck lymph node metastases in our institution between February 2017 and August 2019. Lymph node metastases were excluded if

\footnotetext{
Abbreviations: CT, computed tomography; CTCAE the Common Terminology Criteria for Adverse Events; CTV, clinical target volume; EBER, Epstein-Barr virus-encoded small ribonucleic acid; EBV, Epstein-Barr virus; FDG, fluorodeoxyglucose; GTV, gross tumor volume; HDDP, homogeneous-dose distribution policy; HNSCC, head and neck squamous cell carcinoma; HPV, human papilloma virus; IIHD, intentional internal high dose; IIHDP, intentionally internal high dose policy; IMRT, intensity-modulated Radiotherapy; ISH, in situ hybridization; LRFS, local recurrence free survival; MRI, magnetic resonance imaging; OAR, organ at risk; PET, positron emission tomography; PRV, planning risk volume; PTV, planning target volume; RNA, ribonucleic acid; SIB, simultaneous integrated boost; VMAT, volumetric modulated-arc therapy.
}

they measured $<2 \mathrm{~cm}$ on axial computed tomography (CT)/ magnetic resonance imaging (MRI) images at the initiation of radiotherapy, or if they were irradiated with a prescription dose of more than $2 \mathrm{~Gy}$ in a fraction. Ultimately, 71 lymph node metastases from 51 patients were analyzed in detail. The diagnosis of lymph node metastasis was confirmed by biopsy or based on markedly increased uptake of ${ }^{18} \mathrm{~F}$ fluorodeoxyglucose (FDG) in the area of lymph node metastases on positron emission tomography (PET). In total, 37 lymph node metastases from 25 patients were treated using IIHDP VMAT (group A), while 34 lymph node metastases from 27 patients were treated with homogeneous-dose distribution policy (HDDP) VMAT (group B). One patient with three lymph node metastases had one lymph node assigned to group $\mathrm{A}$ and the others to group B. The following factors were investigated in each group: age, sex, Zubrod performance status, body mass index, hemoglobin at the initiation of radiotherapy, albumin at the initiation of radiotherapy, primary site, the major axis of targeted lymph node metastasis (GTVn), TNM stage (based on the $8^{\text {th }}$ edition of the Union for International Cancer Control TNM staging system), TP53 mutation, postoperative recurrence, Brinkman index, drinking status, virus-associated tumor, concurrent drug administration, prescription radiation dose, radiotherapy durations, number of re-plannings and number of re-plannings due to tumor shrinkage. TNM stage was not investigated in patients with recurrent lymph node metastasis after surgery. Virus-associated tumors were defined as those associated with human papilloma virus (HPV) and Epstein-Barr virus (EBV). To detect HPV, p16 expression was evaluated using pathological specimens: strong, diffuse nuclear and cytoplasmic staining in $70 \%$ or more of the tumor cells was defined as p16 positivity (9). The patients' EBV status was examined using paraffin section RNA in situ hybridization, which uses digoxigenin-labeled riboprobes to visualize EBV-encoded small RNA. Concurrent drug administration status was divided into three groups: chemoradiotherapy (with cisplatin or carboplatin), bioradiotherapy (with cetuximab), and radiotherapy alone.

\section{Treatment Methods}

The slice thickness of the CT images for treatment planning was $2 \mathrm{~mm}$. Non-contrast CTs were used for treatment planning, and delineations were performed with reference to contrast CTs when the patients had no renal dysfunction or allergies to contrast agents.

Gross tumor volume of GTVn was contoured with reference to PET imaging and/or MRI. No clinical target volume margins were set, while the planning target volume (PTV) margins were set to $5 \mathrm{~mm}$ in all directions. When the PTV of the targeted 


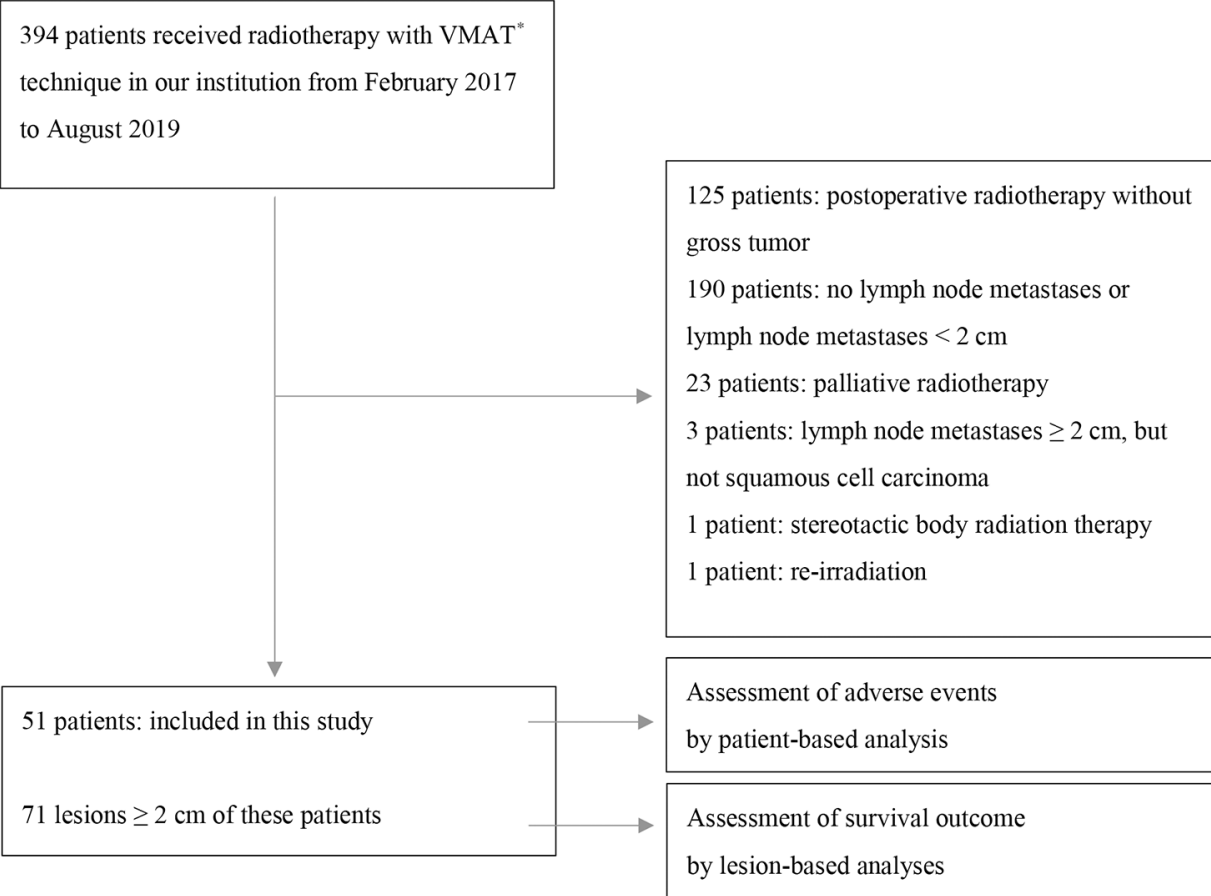

VMAT*, volumetric modulated-arc therapy.

lymph node metastasis (PTVn) extended outside the body, it was cropped to within $0-2 \mathrm{~mm}$ from the body surface. In group A, the IIHD area was contoured inside the lymph node and delivered $110 \%$ to $150 \%$ of the prescription dose as a guide that 1-2 mm needs for decreasing 10\% dose. For example, a $110 \%$ IIHD area was contoured equal to or longer than 1-2 mm inside of GTVn. No PTV margins were set from the IIHD areas. All patients were treated using the VMAT technique at 2 Gy per fraction, once a day and five times per week. Radiotherapy was delivered using $6 \mathrm{MV} \mathrm{X}$-rays from linear accelerators (Varian, Palo Alto, California, USA). Cone-beam CT images were taken more than once a week to allow CT-based guidance. When substantial shrinkage of the tumor or body shape occurred, replanning was performed immediately to make an adaptive treatment plan. When the lymph node metastases had shrunk to less than $2 \mathrm{~cm}$ in diameter under IIHD treatment, the IIHD areas were erased. The IIHDP was applied when the attending physician judged that the lymph node metastases would not achieve complete response with HDDP VMAT, basing this judgment on the primary site and size of GTVn. The prescription dose basically covered $95 \%$ of the PTV. If necessary, the dose coverage on the PTV was reduced to spare OARs, while maximizing coverage as much as possible. Additionally, depending on the distance between the IIHD areas and the mucosal/skin surface, the planning risk volume $(\mathrm{PRV})$ margin of these organs was set to more than $1 \mathrm{~mm}$ in all directions. The frequency of cone-beam image guidance was increased to daily or once every 2 days when the PRV margin was not sufficient. When cone-beam CT was taken, image-guided radiotherapy (IGRT) was subsequently administered based on the IIHD areas, PTV, and OARs. When no cone-beam CT images were taken, radiotherapy was performed based on the bony structures.

\section{Assessment of Outcomes}

The effectiveness was investigated using lesion-based analysis, which accurately evaluates local effectiveness of IIHDP VMAT. Adverse events were evaluated using patient-based analysis because the locations of dermatitis and mucositis were not clear. Dermatitis and mucositis were evaluated by inspection and an endoscopy examination twice a week by two physicians during radiotherapy, and after the termination of radiotherapy, checked at least once a month for one year and after that, at least every 3 months. Acute dermatitis and mucositis were defined as those occurring within 3 months after the completion of radiotherapy. Acute dermatitis and mucositis, as well as late skin disorder and mucosal damage $\geq$ grade 2 , were evaluated using the Common Terminology Criteria for Adverse Events ver. 5.0 in the both groups. Furthermore, we evaluated whether the observed adverse grade 3 effects were associated with IIHDP VMAT, in accordance with NCI guidelines for investigators. Specifically, in group $\mathrm{A}$, we adhered to the adverse events reporting requirements of the NCI Division of Cancer Treatment, including those of the Diagnosis Cancer Therapy Evaluation Program and Cancer Imaging Program, as well as those of the Division of Cancer Prevention, such as Investigational New Drugs and Investigational Device Exemptions (definite, probable, possible, unlikely, unrelated). The one patient with lesions in both groups $\mathrm{A}$ and $\mathrm{B}$ was allocated to group A for adverse events assessment. 
Local recurrence-free survival (LRFS), disease-free survival (DFS), and OS were analyzed for all lesions. Local recurrences of irradiated lymph node metastases were defined as regrowth of the tumor, as was the detection of tumor cells upon biopsy or salvage operation, or markedly increased uptake of ${ }^{18}$ F-FDG in the areas of lymph node metastasis on PET. In case residual tumor was suspected, ${ }^{18}$ F-FDG-PET, CT, or MRI was taken to identify the viability of the tumor. When no tumor cells were detected on salvage operation specimens, the lymph node metastases were considered censored. However, when no residual tumor was detected in lesions that underwent biopsy, follow-up was continued. Addition to LPFS, true LPFS considering salvage surgery (tLPFS) was investigated. In this analysis, when there is no local recurrence after salvage surgery, the lesion is regarded as no local recurrence even if recurrence was detected in the surgical findings.

\section{Statistical Analyses}

The distributions of patient and treatment characteristics were compared between the two groups. To compare categorical variables, the $\chi^{2}$ test was used, and continuous variables were compared using the Mann-Whitney $U$ test. The frequencies of dermatitis and mucositis were compared between the two groups using logistic regression analysis. LRFS, DFS, and OS were estimated using the Kaplan-Meier method and compared using the log-rank test. Additionally, Cox regression analysis of LRFS was performed by applying propensity scores to adjust for differences in characteristics in 2 ways: (1) regression adjustment (2) stratification on the propensity score quintile. P-values $<0.05$ were considered statistically significant. Statistical analyses were performed using IBM SPSS version 26 software (IBM Corp., Armonk, NY, USA).

\section{RESULTS}

\section{Patient/Treatment Characteristics}

Table 2 shows the patient and treatment characteristics by lesion-based grouping. There were 24 nasopharyngeal and 23 oropharyngeal cancer lesions. Fifteen lesions of 10 patients were recurrent lymph node metastases after prior operation. The total number of re-plannings was not significantly different between the two groups, but re-planning due to tumor shrinkage more frequently occurred in group A $(p=0.006)$. The dose-volume histogram analyses are shown in Table 3. The median volumes of the GTVn in groups A and B were $13.4 \mathrm{~cm} 3$ (range: 3.0-423.6 $\mathrm{cm} 3)$ and $7.5 \mathrm{~cm} 3$ (range: $3.1-105.1 \mathrm{~cm} 3)(\mathrm{p}=0.103)$, respectively. The maximal dose of GTVn was significantly higher in group A $(p<0.001)$. GTVn D98\% was significantly higher in group A ( $p=0.018)$, but PTVn D98\% was not significantly different between the two groups $(p=0.067)$.

\section{IIHDP VMAT Treatment in Group A}

The median IIHD volume was $1.7 \mathrm{~cm} 3$ (range: $0.1-76.5 \mathrm{~cm} 3$ ), and the median percentages of the volumes actually irradiated $110 \%$ dose to GTVn volumes were $41.2 \%$ (range: $12.5-92.0 \%$ ).
An example of IIHDP VMAT is shown in Figure 1. IIHDP VMAT was terminated after a median of 19 fractions (range: 733) in 18 patients. The median distances between the margins of the IIHD areas and the mucosal/skin surfaces were $1.95 \mathrm{~cm}$ (range: $0.31-4.92 \mathrm{~cm}$ ) and $1.28 \mathrm{~cm}$ (range: $0.43-4.76 \mathrm{~cm}$ ), respectively. Scheduled radiotherapy was completed in all 28 patients. Grade 3 mucositis unrelated to IIHDP radiotherapy was detected in six patients, while unrelated grade 3 dermatitis was found in one patient, and possibly related dermatitis in two patients.

\section{Assessment of Vessel Complications, Complications With Salvage Surgery, Skin Disorder and Mucosal Damage, and Survival Analysis}

The median follow-up period was 729 days (range: 115-1459 days). The comparison of acute dermatitis and mucositis $\geq$ grade 2 is shown in Table 4. No significant difference was detected between the two groups. No late skin disorders or mucosal damage $\geq$ grade 2 , or vessel complications were observed in any of the 51 patients. Furthermore, all the two patients who received salvage operations in group A experienced no complications. Nonetheless, two of five patients who received salvage operations in group B experienced complications with salvage operations; one patient developed postoperative wound infection, abscess formation, and tissue necrosis, and underwent reconstruction with a pectoralis major musculocutaneous flap, and the other patient suffered tracheal necrosis, fistula formation at the flap anastomosis, and underwent fistula closure with a pectoralis major musculocutaneous flap.

In total, two lesions received salvage surgery in group $A$, and residual tumors were detected in both of them. On the other hand, five lesions received salvage surgery in group $B$, and residual tumors were detected only in the two lesions of them. Additionally, biopsy was performed on the other six lesions in group A, but no residual tumors were found. The LRFS curves of groups A and B are shown in Figure 2. In the log-rank test, the LRFS was significantly longer in group A $(p=0.007)$. Additionally, in the Cox regression analysis of LRFS, with regression adjustment for propensity score and propensity score quintile stratification, an apparent advantage was observed in group A (OR: 0.121, 95\% confidence interval [CI]: 0.024-0.599, $\mathrm{p}=0.010$; OR: 0.125, 95\% CI: 0.026-0.596, $\mathrm{p}=$ $0.009)$. The propensity for receiving IIHDP VMAT was estimated using a multivariable logistic regression model that included baseline GTV volumes and primary site. Furthermore, tLRFS was significantly longer in group $A(p=0.002$, Figure 3 ). In contrast, neither DFS nor OS were significantly different between the two groups ( $\mathrm{p}=0.954,0.939$ ).

\section{DISCUSSION}

In the present study, we investigated the safety and effectiveness of IIHDP VMAT for neck lymph node metastases of HNSCC that measured $\geq 2 \mathrm{~cm}$. In patients who received IIHDP VMAT, 
TABLE 2 | Patient and treatment characteristics in group A and B with lesion-based grouping (71 neck lymph nodes).

\begin{tabular}{|c|c|c|c|}
\hline Parameters & $\begin{array}{c}\text { Group A (37 lesions) } \\
\text { n (\%) }\end{array}$ & $\begin{array}{c}\text { Group B (34 lesions) } \\
\text { n (\%) }\end{array}$ & $p$ value \\
\hline Age, median (range) & $63(8-83)$ & $63(32-84)$ & 0.549 \\
\hline Sex & & & 0.823 \\
\hline Male & $27 / 37(73.0 \%)$ & $24 / 34(70.6 \%)$ & \\
\hline Female & $10 / 37(27.0 \%)$ & $10 / 34(29.4 \%)$ & \\
\hline Zubrod Performance status & & & 0.376 \\
\hline 0 & $26 / 37(70.3 \%)$ & $27 / 34(79.4 \%)$ & \\
\hline 1 & $11 / 37(29.7 \%)$ & $7 / 34(20.6 \%)$ & \\
\hline Body mass index & $20.5(11.8-23.9)$ & $19.8(14.3-28.5)$ & 0.454 \\
\hline Hemoglobin at the initiation of radiotherapy median (range) & $13.7(8.8-16.4)$ & $13.8(11.2-17.8)$ & 0.991 \\
\hline Albumin at the initiation of radiotherapy median (range) & $4.0(3.3-4.8)$ & $4.2(3.3-4.8)$ & 0.175 \\
\hline Primary site & & & 0.429 \\
\hline Nasopharynx & $9 / 37$ (24.3\%) & $15 / 34(44.1 \%)$ & 0.078 \\
\hline Oropharynx & $14 / 37(37.8 \%)$ & $9 / 34(26.5 \%)$ & 0.307 \\
\hline Hypopharynx & $6 / 37(16.2 \%)$ & $5 / 34(14.7 \%)$ & 0.861 \\
\hline Larynx & $2 / 37(5.4 \%)$ & $1 / 34(2.9 \%)$ & 0.606 \\
\hline Oral cavity & 6/37 (16.2\%) & $3 / 34(8.8 \%)$ & 0.350 \\
\hline Maxillary sinus & 0/37 (0.0\%) & $1 / 34(2.9 \%)$ & 0.293 \\
\hline The major axis diameter of GTVn median (range) & $27(20-82)$ & $25(20-70)$ & 0.073 \\
\hline \multicolumn{4}{|c|}{ TNM stage (without recurrent patients, 27 and 29 lesions, respectively) } \\
\hline T stage & & & 0.225 \\
\hline 1 & $6 / 27(22.2 \%)$ & $9 / 29(31.0 \%)$ & \\
\hline 2 & $14 / 27(51.9 \%)$ & $8 / 29(27.6 \%)$ & \\
\hline 3 & $3 / 27(11.1 \%)$ & $5 / 29(17.2 \%)$ & \\
\hline 4 & $4 / 27(14.8 \%)$ & $7 / 29(24.1 \%)$ & \\
\hline N stage & & & 0.588 \\
\hline 1 & $8 / 27(29.6 \%)$ & $10 / 29(34.5 \%)$ & \\
\hline 2 & $13 / 27(48.1 \%)$ & $11 / 29(37.9 \%)$ & \\
\hline 3 & $6 / 27(22.2 \%)$ & $8 / 29(27.6 \%)$ & \\
\hline TP53 mutation, number & & & 0.543 \\
\hline Wild type & $21 / 37(56.8 \%)$ & $18 / 34(52.9 \%)$ & \\
\hline Mutation type & $12 / 37(32.4 \%)$ & $14 / 34(41.2 \%)$ & \\
\hline Unknown & $4 / 37(10.8 \%)$ & $2 / 34(5.9 \%)$ & \\
\hline Postoperative recurrence & $9 / 37(24.3 \%)$ & 6/34 (17.6\%) & 0.491 \\
\hline $\mathrm{Bl}^{\star}$, median (range) & $200(0-1740)$ & $80(0-1200)$ & 0.273 \\
\hline Drinking status, number & & & 0.256 \\
\hline Yes & 22/37 (59.5\%) & 23/34 (67.6\%) & \\
\hline No & $14 / 37(37.8 \%)$ & 8/34 (23.5\%) & \\
\hline Unknown & $1 / 37(2.7 \%)$ & 3/34 (8.8\%) & \\
\hline Virus associated & $20 / 37(54.1 \%)$ & $22 / 34(64.7 \%)$ & 0.362 \\
\hline Concurrent drug administration & & & 0.106 \\
\hline Chemoradiotherapy, number & 35/37 (94.6\%) & 27/34 (79.4\%) & \\
\hline Bioradiotherapy, number & $1 / 37(2.7 \%)$ & 6/34 (17.6\%) & \\
\hline Radiotherapy alone, number & $1 / 37(2.7 \%)$ & $1 / 34(2.9 \%)$ & \\
\hline Prescription radiation dose & & & 0.627 \\
\hline 70 Gy in 35 fractions & 35/37 (94.6\%) & 33/34 (97.1\%) & \\
\hline 66 Gy in 33 fractions & $1 / 37(2.7 \%)$ & 0/34 (0.0\%) & \\
\hline 60 Gy in 30 fractions & $1 / 37(2.7 \%)$ & $1 / 34(2.9 \%)$ & \\
\hline Radiotherapy durations (days), median (range) & $52(46-61)$ & $51(50-55)$ & 0.064 \\
\hline With re-planning & $29 / 37(78.4 \%)$ & $33 / 34(97.1 \%)$ & 0.445 \\
\hline With re-planning due to tumor shrinkage & $15 / 37(40.5 \%)$ & 4/34 (11.8\%) & 0.006 \\
\hline
\end{tabular}

Bl, Brinkman index.

Bolded text means that they have a statistically significant meaning.

LRFS was significantly longer, with no increase in adverse effects. This result was consistent after regression adjustment for propensity score and propensity score quintile stratification.

IIHDP radiotherapy was previously described by Kashihara et al. (8), who reported the case of a bulky uterine cervical neoplasm that was irradiated using IIHDP brachytherapy. The transverse diameter at the initiation of brachytherapy was $8.9 \mathrm{~cm}$, but complete response was eventually obtained after treatment. Additionally, in treating bulky hepatocellular carcinoma, simultaneous integrated boost (SIB) with simultaneous integrated protection technique was reported by Crane et al. (10) This technique also applied dose escalation to tumors while protecting OARs. A retrospective analysis in patients treated using a similar technique for inoperable intrahepatic cholangiocarcinoma was reported by Tao et al. (11) In inoperable intrahepatic cholangiocarcinoma, improvements in local control and OS was observed when higher doses were delivered. On the other hand, in HNSCC patients, GORTEC 
TABLE 3 | Dose-volume histogram analyses in group A and B.

\begin{tabular}{|c|c|c|c|}
\hline Parameters, median (range) & Group A & Group B & $p$ value \\
\hline GTVn* volume (cc) & $13.0(3.0-423.6)$ & $4.5(3.1-105.1)$ & 0.103 \\
\hline $\mathrm{IHD}^{\dagger}$ area volume $(\mathrm{cc})$ & $1.7(0.1-76.5)$ & 0 & $\mathrm{~N} / \mathrm{A}$ \\
\hline GTVn max (\%) & $124.0(112.2-173.9)$ & $107.9(104.9-111.4)$ & $<0.001$ \\
\hline GTVn D98\% (\%) & $102.7(100.7-108.0)$ & $102.3(98.0-104.2)$ & 0.018 \\
\hline PTVn D98\% (\%) & $101.3(99.4-104.0)$ & $102.0(93.4-103.6)$ & 0.067 \\
\hline PTVn D50\% (\%) & 105.6 (104.2-115.2) & 104.7 (100.3-107.2) & $<0.001$ \\
\hline PTVn RTOG ${ }^{\S}$ Homogeneity index & $1.240(1.122-1.739)$ & $1.093(1.065-1.125)$ & $<0.001$ \\
\hline PTVn ICRU' Homogeneity index & 0.179 (0.0733-0.6243) & 0.0468 (0.0327-0.122) & $<0.001$ \\
\hline
\end{tabular}

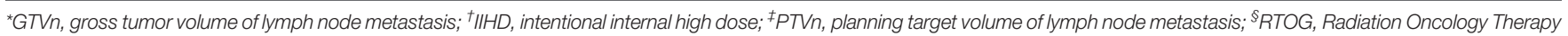
Group; IICRU, International Commission on Radiation Units and Measurements.

Bolded text means that they have a statistically significant meaning.

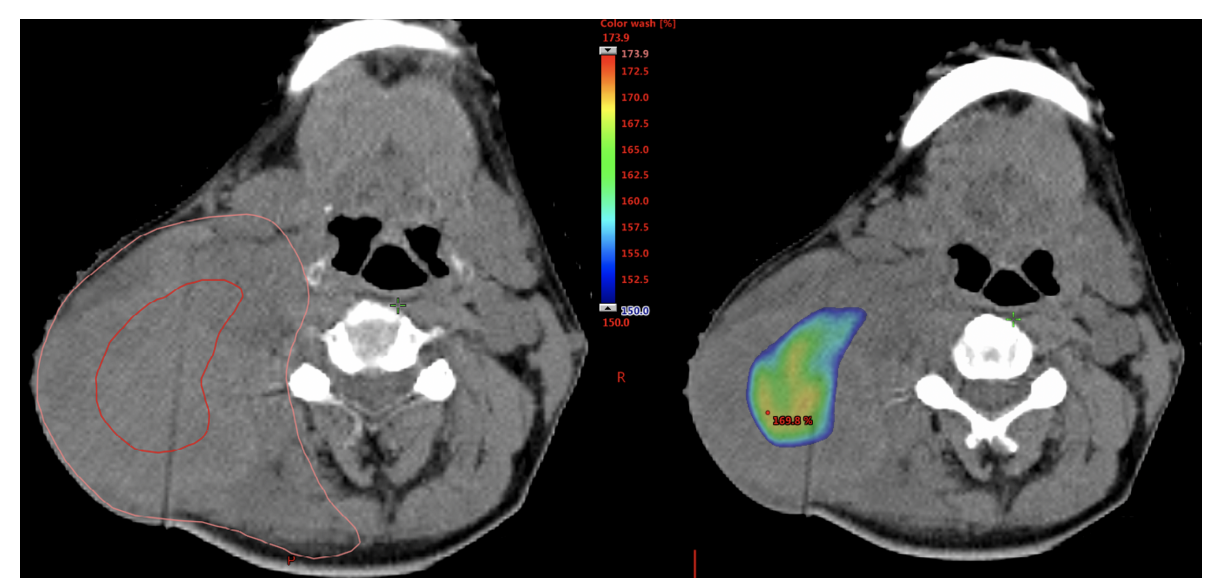

FIGURE 1 | Example of the dose distribution of intentionally internal high dose policy (IIHDP) volumetric modulated arc therapy (VMAT) to treat nasopharyngeal cancer with massive neck lymph node metastases. In the left picture, delineations with red and orange lines represent intentionally internal high dose (IIHD) area and the clinical target volume (CTV), respectively. In the right picture, dose $\geq 150 \%$ of the prescription dose are shown as dose color wash.

TABLE 4 | Comparison of acute dermatitis and mucositis in group A and B (Patient-based analyses).

\begin{tabular}{|c|c|c|c|c|}
\hline Parameters & n (\%) & Odds ratio & $95 \%$ confidence interval & $p$ value \\
\hline \multicolumn{5}{|c|}{ Dermatitis $\geq$ grade 2} \\
\hline Group A & $15 / 25(60.0 \%)$ & 0.553 & $0.170-1.798$ & 0.324 \\
\hline Group B & 19/26 (73.1\%) & 1.000 (reference) & & \\
\hline \multicolumn{5}{|c|}{ Dermatitis $\geq$ grade 3} \\
\hline Group A & $3 / 25$ (12.0\%) & 0.573 & $0.121-2.702$ & 0.481 \\
\hline Group B & $5 / 26(19.2 \%)$ & 1.000 (reference) & & \\
\hline \multicolumn{5}{|c|}{ Mucositis $\geq$ grade 2} \\
\hline Group A & $15 / 25$ (60.0\%) & 0.450 & $0.134-1.514$ & 0.197 \\
\hline Group B & 20/26 (76.9\%) & 1.000 (reference) & & \\
\hline \multicolumn{5}{|c|}{ Mucositis $\geq$ grade 3} \\
\hline Group A & $6 / 25$ (24.0\%) & 1.053 & $0.289-3.840$ & 0.938 \\
\hline Group B & 6/26 (23.1\%) & 1.000 (reference) & & \\
\hline
\end{tabular}

2004-01 randomized phase III trial reported that dose-escalated IMRT did not decrease locoregional recurrence in comparison with $70 \mathrm{~Gy}$ in 35 fractions (7). In dose-escalated IMRT group, a sequential boost of 25 Gy in 10 fractions to initial GTV was delivered after 50 Gy in 25 fractions to prophylactic PTV. Compared with this method, higher dose can be irradiated to the center of the tumors with IIHDP VMAT. Calculating the biologically effective dose to the center of the tumors with $\alpha / \beta=$ $10 \mathrm{~Gy}$, the dose-escalated IMRT technique in GORTEC 2004-01 trial is $81 \mathrm{~Gy}$, and IIHDP VMAT is $94 \mathrm{~Gy}(110 \%)$ to $137 \mathrm{~Gy}$ (150\%). Therefore, IIHDP VMAT could be more effective technique for improving local control of the tumors.

Some previous studies have reported dose escalation using a VMAT technique in head and neck cancer (7, 12-17). Lauve et al. reported the results of the phase I trial of dose escalation to GTV in bulky HNSCC patients (12). Of 12 patients at dose level 2 (70.8 Gy in 30 fractions), 4 patients had Grade 3 acute skin toxicities, 6 had Grade 3 acute dysphagia, and 8 had Grade 3 


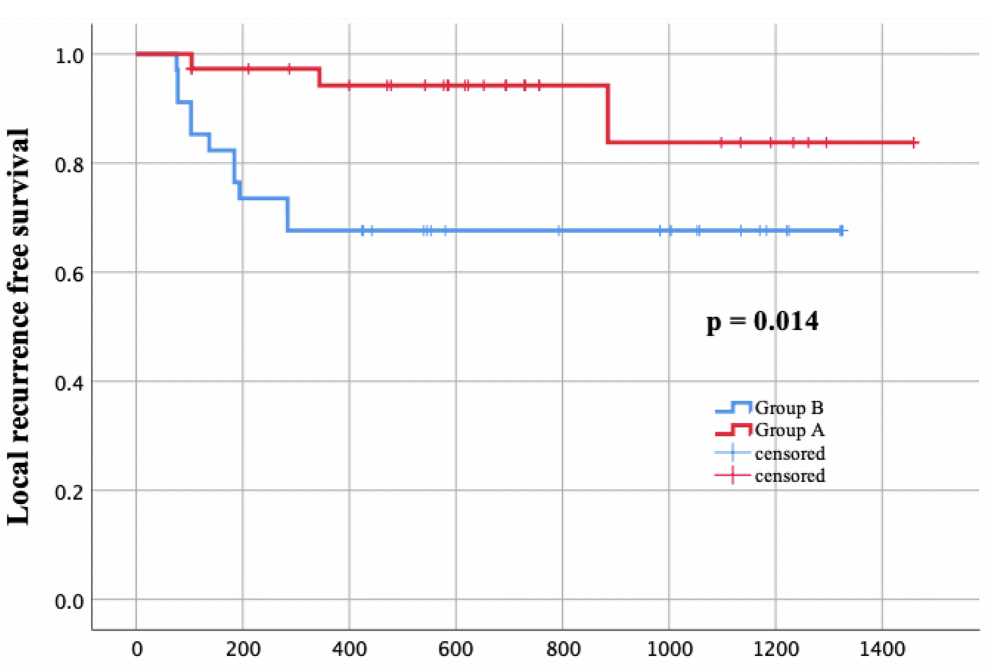

No. at Risk

Days without local recurrence from the initiation of radiotherapy

$\begin{array}{rllllllll}\text { Group A } & 37 & 34 & 30 & 22 & 9 & 8 & 5 & 2 \\ \text { B } & 34 & 25 & 23 & 16 & 15 & 13 & 5 & 0\end{array}$

FIGURE 2 | Local recurrence free survival (LRFS) in groups A (red line) and B (blue line).

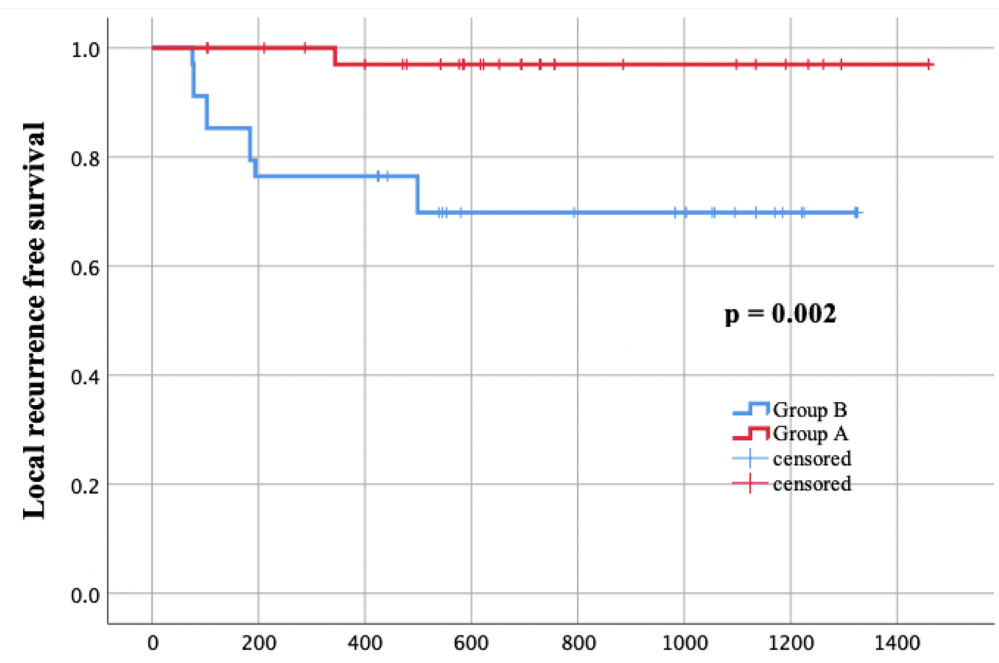

No. at Risk

Days without local recurrence from the initiation of radiotherapy Group A

$\begin{array}{llllllll}37 & 35 & 31 & 23 & 10 & 9 & 6 & 2 \\ 34 & 26 & 26 & 17 & 16 & 14 & 5 & 0\end{array}$

FIGURE 3 | Local recurrence free survival considering salvage surgery (tLRFS) in groups A (red line) and B (blue line).

acute mucositis. Furthermore, at dose level 3 (73.8 Gy in 30 fractions), radiotherapy was suspended due to premature Grade 3 dysphagia and mucositis in both of the 2 patients. Additionally, a Phase I/II study reported that $87 \%$ of the patients who received 67.2 Gy in 28 fractions for PTV had Grade 3 dysphagia (13). Dose escalation using HDDP VMAT could increase serious adverse effects because the definitions of irradiation increase the dose outside of the GTV and could increase the dose outside of the PTV. In the PET-guided focal-dose escalation study for HNSCC patients (14), although this technique was quite reasonable for improving tumor control, two patients experienced grade 4 adverse effects (one patient: dermatitis and the other: dysphagia) and one treatment related death was reported. In this study, PTV D98\% was about 106\% in dose level I and $111 \%$ of prescription dose in dose level II. On the other hand, in our study, PTVn D98\% was not significantly 
increased (median, $101.3 \%$ in group A), indicating that the peripheral dose of PTVn area was not increased by using IIHDP VMAT (Table 3). This analysis means that OARs outside of the PTVn can be protected using IIHDP VMAT. However, GTVn D98\% was significantly higher in group A, thus OARs inside of the PTVn may require more attention than was previously thought. No increase in adverse effects, such as dermatitis and mucositis, with the application of IIHDP was observed in the present study, but to increase the safety of this irradiation method, the GTVn D98\% should be taken into consideration.

In many cases of bulky neck lymph node with a favorable response to radiotherapy, the inside of the tumor becomes necrotic and viable, contrast-enhanced lesions remain in the peripheral part of the bulky lymph nodes. In such circumstances, it is unknown why increasing the inner dose can contribute to controlling the bulky tumors, not by increasing the peripheral dose, but it might be related to hypoxia. Large tumors are associated with hypoxia inside of the tumor, which can weaken the effect of radiotherapy (18). In a randomized phase II trial of dose escalation using dynamic ${ }^{18}$ F-fluoromisonidazole PET/CT for locally advanced HNSCC patients, locoregional control was worse in patients with hypoxic tumors (15). An animal model has indicated that high dose irradiation to the hypoxic area inside of the tumor led to better local control (19). Additionally, RA Popple et al. reported that a modest boost dose (120-150\% dose) to temporary hypoxic area would increase tumor control probability (20). It follows that IIHDP VMAT may be a reasonable technique for enhancing local control. Moreover, it is one of the strengths of IIHDP VMAT that no special image inspections are required such as ${ }^{18} \mathrm{~F}$-FDG-PET/CT or ${ }^{18}$ F-fluoromisonidazole PET/CT.

In the patient whose one lymph node metastasis with a major axis diameter of $30 \mathrm{~mm}$ (lesion A) was treated using IIHDP VMAT (group A) and two lymph node metastases with major axes of 22 and $21 \mathrm{~mm}$ (lesion $\mathrm{B}_{1}$ and $\mathrm{B}_{2}$ ) were irradiated with HDDP VMAT (group B), complete response was seen in lesion $\mathrm{A}$, while partial response was seen in lesions $\mathrm{B}_{1}$ and $\mathrm{B}_{2}$. Ten months after the completion of radiotherapy, ${ }^{18} \mathrm{~F}-\mathrm{FDG}-$ PET MRI was taken to detect recurrent lesions. No increased uptake of ${ }^{18}$ F-FDG was seen in lesion $A$, but markedly increased uptake of ${ }^{18} \mathrm{~F}$-FDG was seen in lesions $\mathrm{B}_{1}$ and $\mathrm{B}_{2}$, indicating that the IIHDP had been effective. Additionally, while the DFS was not related to IIHDP, the LRFS was significantly longer in group A, suggesting that IIHDP had local effectiveness.

The patient and treatment characteristics between the two groups were well-balanced, but there were marginally more patients with nasopharyngeal cancer in group B. Metastases from nasopharyngeal carcinoma are more likely to be controlled than those of other HNSCCs $(21,22)$. Despite this, local control of lymph node metastases was better in group A. Furthermore, nodal size and primary site are significant predictive factors of definitive radiotherapy in oropharyngeal and pharyngolaryngeal cancers $(23,24)$.
Hence, these two factors were used to adjust patient characteristics with propensity score, and the results were consistent before and after the adjustment.

Regarding the number of re-plannings, no significant difference was found between the two groups. However, replannings due to tumor shrinkage were performed significantly more times in group A. This result implies that IIHDP VMAT conferred better tumor shrinkage during radiotherapy, leading to better LRFS in group A. To confirm this finding, additional research is needed.

In previous reports of head and neck cancer, IMRT using a SIB technique did not improve local control compared to $3 \mathrm{D}$ conformal radiotherapy and IMRT without a SIB technique, even though the PTV coverage was superior $(25,26)$. Likewise, in nasopharyngeal carcinoma, IMRT improved quality of life, but did not improve survival outcomes compared to $3 \mathrm{D}$ conformal radiotherapy (27). To the best of our knowledge, this was the first report on the effectiveness of dose escalation using a SIB-VMAT technique to treat neck lymph node metastases of HNSCC.

There were two limitations in the present study. Firstly, we could not evaluate whether mucosal dose and adverse effects increased or not by using IIHDP VMAT because a high dose was irradiated onto the primary tumor close to the metastatic lymph node. Nonetheless, the total number of mucositis cases with $\geq$ grade 2 or 3 was not larger in group A than in group B. In the present study, the IIHD area was contoured longer than $3 \mathrm{~mm}$ from the mucosal surface, so it is unlikely that any high dose was irradiated onto the mucosa. It can be used as a guide of a safe distance from the IIHD area to OARs. Secondly, this report was retrospective, with a small number of patients and a short follow-up period, thus it may have included some unknown biases. Especially, group A consists of patients who were selected at the discretion of the attending physician based on the lymph node size and primary site, thus this method introduces major selection bias. Additionally, most of the patients in group A received concurrent chemoradiotherapy, but in group B, only $79 \%$ of the patients received concurrent chemoradiotherapy, although the difference was not statistically significant. This difference could contribute to the better local control rate in group A. Moreover, because of a lack of standard dose escalation method, this study could be difficult to reproduce in other studies. Further additional research, including prospective trials that clearly defines the method of IIHDP to be reproducible, should solve this problem. The method would be to set the contouring of $110 \%$ IIHDP area $3-5 \mathrm{~mm}$ inside the GTV, the contouring of $120 \%$ IIHDP area $3-5 \mathrm{~mm}$ inside the $110 \%$ IIHDP area, increasing the internal dose by $10 \%$ every $3-5 \mathrm{~mm}$ up to $150 \%$. Furthermore, the locoregional recurrence survival should be assessed using tLPFS, which does not count as a recurrence if it can be salvaged by surgery, and it should be verified whether a dose escalation with IIHDP VMAT in a larger number of patients could outweigh the toxicity of selective neck dissection in patients without complete response with HDDP VMAT. 


\section{CONCLUSION}

Our initial experience of IIHDP VMAT suggested that IIHDP VMAT to treat HNSCC neck lymph node metastases measuring $\geq 2 \mathrm{~cm}$ was feasible and possibly led to better local control than HDDP VMAT. To verify the effectiveness of IIHDP VMAT, a large-scale prospective study that clearly defines the method of IIHDP to be reproducible would be required.

\section{DATA AVAILABILITY STATEMENT}

The raw data supporting the conclusions of this article will be made available by the authors, without undue reservation.

\section{ETHICS STATEMENT}

The studies involving human participants were reviewed and approved by The Institutional Review Board of the National

\section{REFERENCES}

1. Bray F, Ferlay J, Soerjomataram I, Siegel RL, Torre LA, Jemal A. Global cancer statistics 2018: GLOBOCAN estimates of incidence and mortality worldwide for 36 cancers in 185 countries. CA Cancer J Clin (2018) 68(6):394-424. doi: $10.3322 /$ caac. 21492

2. Marta GN, Silva V, de Andrade Carvalho H, de Arruda FF, Hanna SA, Gadia R, et al. Intensity-modulated radiation therapy for head and neck cancer: systematic review and meta-analysis. Radiother Oncol (2014) 110 (1):9-15. doi: 10.1016/j.radonc.2013.11.010

3. Rathod S, Gupta T, Ghosh-Laskar S, Murthy V, Budrukkar A, Agarwal J. Quality-of-life (QOL) outcomes in patients with head and neck squamous cell carcinoma (HNSCC) treated with intensity-modulated radiation therapy (IMRT) compared to three-dimensional conformal radiotherapy (3D-CRT): evidence from a prospective randomized study. Oral Oncol (2013) 49(6):63442. doi: 10.1016/j.oraloncology.2013.02.013

4. Pow EH, Kwong DL, McMillan AS, Wong MC, Sham JS, Leung LH, et al. Xerostomia and quality of life after intensity-modulated radiotherapy vs. conventional radiotherapy for early-stage nasopharyngeal carcinoma: initial report on a randomized controlled clinical trial. Int J Radiat Oncol Biol Phys (2006) 1566(4):981-91. doi: 10.1016/j.ijrobp.2006.06.013

5. McMillan AS, Pow EH, Kwong DL, Wong MC, Sham JS, Leung LH, et al. Preservation of quality of life after intensity-modulated radiotherapy for early-stage nasopharyngeal carcinoma: results of a prospective longitudinal study. Head Neck (2006) 28(8):712-22. doi: 10.1002/hed.20378

6. Parliament MB, Scrimger RA, Anderson SG, Kurien EC, Thompson HK, Field GC, et al. Preservation of oral health-related quality of life and salivary flow rates after inverse-planned intensity- modulated radiotherapy (IMRT) for head-and-neck cancer. Int J Radiat Oncol Biol Phys (2004) 158(3):663-73. doi: 10.1016/S0360-3016(03)01571-2

7. Tao Y, Auperin A, Blanchard P, Alfonsi M, Sun XS, Rives M, et al. Concurrent cisplatin and dose escalation with intensity-modulated radiotherapy (IMRT) versus conventional radiotherapy for locally advanced head and neck squamous cell carcinomas (HNSCC): GORTEC 2004-01 randomized phase III trial. Radiother Oncol (2020) 150:18-25. doi: 10.1016/j.radonc.2020.05.021

8. Kashihara T, Kobayashi K, Iijima K, Murakami N, Yoshida K, Okuma K, et al. A case report of a patient with bulky uterine cervical neoplasm who achieved complete response with "intentional internal high-dose policy" high-doserate interstitial brachytherapy. Med (Baltimore) (2020) 299(27):e20860. doi: 10.1097/MD.0000000000020860
Cancer Center Hospital (approval number, 2017-091). The patients/participants provided their written informed consent to participate in this study. Written informed consent was obtained from the individual(s) for the publication of any potentially identifiable images or data included in this article.

\section{AUTHOR CONTRIBUTIONS}

Conception and design of the work: TK, SN, NM, KiI, YM, KKa, GO, TM, YH, YK, HO, KT, KoI, KO, HI, YN, KKo, FM, SY, JI. Supervision and wrote the paper: TK. All authors contributed to the article and approved the submitted version.

\section{ACKNOWLEDGEMENTS}

We would like to thank Editage (www.editage.jp) for English language editing and Ryunosuke Machida for advice on statistical analyses.

9. Begum S, Gillison ML, Ansari-Lari MA, Shah K, Westra WH. Detection of human papillomavirus in cervical lymph nodes: a highly effective strategy for localizing site of tumor origin. Clin Cancer Res (2003) 159 (17):6469-75.

10. Crane $\mathrm{CH}$, Koay EJ. Solutions that enable ablative radiotherapy for large liver tumors: Fractionated dose painting, simultaneous integrated protection, motion management, and computed tomography image guidance. Cancer (2016) 1122(13):1974-86. doi: 10.1002/cncr.29878

11. Tao R, Krishnan S, Bhosale PR, Javle MM, Aloia TA, Shroff RT, et al. Ablative Radiotherapy Doses Lead to a Substantial Prolongation of Survival in Patients With Inoperable Intrahepatic Cholangiocarcinoma: A Retrospective Dose Response Analysis. J Clin Oncol (2016) 2034(3):219-26. doi: 10.1200/ JCO.2015.61.3778

12. Lauve A, Morris M, Schmidt-Ullrich R, Wu W, Mohan R, Abayomi O, et al. Simultaneous integrated boost intensity-modulated radiotherapy for locally advanced head-and-neck squamous cell carcinomas: II-clinical results. Int J Radiat Oncol Biol Phys (2004) 160(2):374-87. doi: 10.1016/j.ijrobp.2004.03.010

13. Miah AB, Bhide SA, Guerrero-Urbano MT, Clark C, Bidmead AM, St Rose S, et al. Dose-escalated intensity-modulated radiotherapy is feasible and may improve locoregional control and laryngeal preservation in laryngohypopharyngeal cancers. Int J Radiat Oncol Biol Phys (2012) 182(2):539-47. doi: 10.1016/j.ijrobp.2010.09.055

14. Madani I, Duthoy W, Derie C, De Gersem W, Boterberg T, Saerens M, et al. Positron emission tomography-guided, focal-dose escalation using intensitymodulated radiotherapy for head and neck cancer. Int J Radiat Oncol Biol Phys (2007) 168(1):126-35. doi: 10.1016/j.ijrobp.2006.12.070

15. Welz S, Mönnich D, Pfannenberg C, Nikolaou K, Reimold M, La Fougère C, et al. Prognostic value of dynamic hypoxia PET in head and neck cancer: Results from a planned interim analysis of a randomized phase II hypoxiaimage guided dose escalation trial. Radiother Oncol (2017) 124(3):526-32. doi: 10.1016/j.radonc.2017.04.004

16. Gujral DM, Miah AB, Bodla S, Richards TM, Welsh L, Schick U, et al. Final long-term results of a phase I/II study of dose-escalated intensity-modulated radiotherapy for locally advanced laryngo-hypopharyngeal cancers. Oral Oncol (2014) 50(11):1089-97. doi: 10.1016/j.oraloncology.2014.07.018

17. Atwell D, Elks J, Cahill K, Hearn N, Vignarajah D, Lagopoulos J, et al. A Review of Modern Radiation Therapy Dose Escalation in Locally Advanced Head and Neck Cancer. Clin Oncol (R Coll Radiol) (2020) 32(5):330-41. doi: 10.1016/j.clon.2019.12.004 
18. Hockel M, Schlenger K, Aral B, Mitze M, Schaffer U, Vaupel P. Association between tumor hypoxia and malignant progression in advanced cancer of the uterine cervix. Cancer Res (1996) 156(19):4509-15.

19. Epel B, Maggio MC, Barth ED, Miller RC, Pelizzari CA, Krzykawska-Serda M, et al. Oxygen-Guided Radiation Therapy. Int J Radiat Oncol Biol Phys (2019) 15103(4):977-84. doi: 10.1016/j.ijrobp.2018.10.041

20. Popple RA, Ove R, Shen S. Tumor control probability for selective boosting of hypoxic subvolumes, including the effect of reoxygenation. Int J Radiat Oncol Biol Phys (2002) 154(3):921-7. doi: 10.1016/S0360-3016(02)03007-9

21. Chow E, Payne D, Keane T, Panzarella T, Izard MA. Enhanced control by radiotherapy of cervical lymph node metastases arising from nasopharyngeal carcinoma compared with nodal metastases from other head and neck squamous cell carcinomas. Int J Radiat Oncol Biol Phys (1997) 139(1):14954. doi: 10.1016/S0360-3016(97)00313-1

22. Ohara K, Tatsuzaki H, Kurosaki Y, Fuji H, Myo-Min, Itai Y, et al. Metastatic lymph-node clearance from head and neck epidermoid carcinomas following radiotherapy. Acta Oncol (1999) 38(2):261-6. doi: 10.1080/028418699431708

23. Bataini JP, Bernier J, Jaulerry C, Brunin F, Pontvert D, Lave C. Impact of neck node radioresponsiveness on the regional control probability in patients with oropharynx and pharyngolarynx cancers managed by definitive radiotherapy. Int J Radiat Oncol Biol Phys (1987) 13(6):817-24. doi: 10.1016/0360-3016(87)90093-9

24. Chao KS, Ozyigit G, Blanco AI, Thorstad WL, Deasy JO, Haughey BH, et al. Intensity-modulated radiation therapy for oropharyngeal carcinoma: impact of tumor volume. Int J Radiat Oncol Biol Phys (2004) 159(1):43-50. doi: 10.1016/j.ijrobp.2003.08.004

25. Spiotto MT, Weichselbaum RR. Comparison of 3D confromal radiotherapy and intensity modulated radiotherapy with or without simultaneous integrated boost during concurrent chemoradiation for locally advanced head and neck cancers. PloS One (2014) 89(4):e94456. doi: 10.1371/journal.pone.0094456
26. Jirkovska M, Novak T, Malinova B, et al. Three-dimensional conformal radiotherapy versus intensity modulated radiotherapy with simultaneous integrated boost in the treatment of locally advanced head and neck carcinoma. Neoplasma (2019) 1966(5):830-8. doi: 10.4149/neo_ 2018_181209N941

27. Fang FM, Chien CY, Tsai WL, et al. Quality of life and survival outcome for patients with nasopharyngeal carcinoma receiving three-dimensional conformal radiotherapy vs. intensity-modulated radiotherapy-a longitudinal study. Int J Radiat Oncol Biol Phys (2008) 172(2):356-64.

Conflict of Interest: YN reports personal fees from AstraZeneca, outside the submitted work. YK reports grants from Canon Medical Systems, outside the submitted work. KI reports grants from Elekta K.K., outside the submitted work HI reports personal fees from Itochu, personal fees from ViewRay Inc., grants from HekaBio, outside the submitted work. JI reports grants and non-financial support from KeyJ, personal fees from Alpha Tau, personal fees from ItoChu, outside the submitted work.

The remaining authors declare that the research was conducted in the absence of any commercial or financial relationships that could be construed as a potential conflict of interest.

Copyright (C) 2021 Kashihara, Nakamura, Murakami, Ito, Matsumoto, Kobayashi, Omura, Mori, Honma, Kubo, Okamoto, Takahashi, Inaba, Okuma, Igaki, Nakayama, Kato, Matsumoto, Yoshimoto and Itami. This is an open-access article distributed under the terms of the Creative Commons Attribution License (CC BY). The use, distribution or reproduction in other forums is permitted, provided the original author(s) and the copyright owner(s) are credited and that the original publication in this journal is cited, in accordance with accepted academic practice. No use, distribution or reproduction is permitted which does not comply with these terms. 